\title{
Detecting the breakup of spiral waves in small-world networks of neurons due to channel block
}

\author{
MA Jun ${ }^{1,2 *}$, HUANG Long ${ }^{1}$, YING HePing ${ }^{3} \&$ PU ZhongSheng ${ }^{1}$ \\ ${ }^{1}$ Department of Physics, Lanzhou University of Technology, Lanzhou 730050, China; \\ ${ }^{2}$ Key Laboratory of Gansu Advanced Control for Industrial Processes, Lanzhou 730050, China; \\ ${ }^{3}$ Zhejiang Institute of Modern Physics, Zhejiang University, Hangzhou 310027, China
}

Received October 26, 2011; accepted January 4, 2012

\begin{abstract}
The breakup of a spiral wave by blockade of sodium and potassium channels in a small-world network of Hodgkin-Huxley neurons is investigated in detail. The influence of ion channel block in poisoned excitable membrane patches of a certain size is measured, by varying channel noise and channel densities resulting from the change in conductance. For example, tetraethylammonium is known to cause a block (poisoning) of potassium channels, while tetrodotoxin blocks sodium channels. We observed the occurrence of spiral waves, which are ordered waves believed to play an important role in facilitating the propagation of electric signals across quiescent regions of the brain. In this paper, the effect of channel block was measured by the factors $x_{\mathrm{K}}$ and $x_{\mathrm{Na}}$, which represent the ratios of unblocked, or active, ion channels, to the overall number of potassium or sodium ion channels, respectively. To quantify these observations, we use a simple but robust synchronization measure, which succinctly captures the transition from spiral waves to other collective states, such as broken segments resulting from the breakup of the spiral wave. The critical thresholds of channel block can be inferred from the abrupt changes occurring in plots of the synchronization measure against different values of $x_{\mathrm{K}}$ and $x_{\mathrm{Na}}$. Notably, small synchronization factors can be tightly associated with states where the formation of spiral waves is robust to mild channel block.
\end{abstract}

spiral wave, factor of synchronization, Hodgkin-Huxley neurons, small-world networks, channel block

Citation: $\quad$ Ma J, Huang L, Ying H P, et al. Detecting the breakup of spiral waves in small-world networks of neurons due to channel block. Chin Sci Bull, 2012, 57: 2094-2101, doi: 10.1007/s11434-012-5114-2

A spiral wave is a characteristic spatiotemporal pattern that is often observed in excitable media [1-10]. The formation and propagation of spiral waves in reaction-diffusion systems have been studied extensively [11-16], and some effective schemes have been used to remove spiral waves and prevent ventricular fibrillation [17]. Stable rotating spiral waves have been observed in rat neocortical slices visualized by voltage-sensitive dye imaging $[18,19]$. Spiral waves might serve as emergent population pacemakers, generating periodic activity in non-oscillatory networks without individual cellular pacemakers. The formation, death, and breakup of spiral waves in the mammalian cortex can be simulated using regular and/or small-world networks [8-10].

*Corresponding author (email: hyperchaos@163.com)
In networks using Hodgkin-Huxley model neurons [20], the effects of channel noise [21,22] on spiral waves needs to be studied in detail.

Normal signal propagation between neurons in networks with different topologies deeply depends on the collective behavior of neurons. Abnormalities of neurons in one domain can destroy the normal communication between neurons, and neurological disorders can occur when the normal electrical activity of neurons is violated. The response of neurons to external stimuli (drugs or electrical forcing) is often a result of the collective behaviors of all the neurons in one or more domains. It is reliable to study the collective electrical activities of neurons in functional domains using complex network schemes. Most previous schemes have been proposed to remove spiral waves in reaction-diffusion 
systems, with the expectation that they may provide reliable ways to prevent ventricular fibrillation. However, spiral waves observed in neocortical slices have been found to play an active role in preserving normal communication in quiescent domains of the neuronal system. It is therefore critical to study the robustness and transition of spiral waves in neocortical slices using complex network schemes. Some interesting studies have shown that ordered waves in neuronal networks could be relative to coherence resonance, and the state could be changed by time delay or other bifurcation parameter shifts $[11,23,24]$. Channel noise in Hodgkin-Huxley neurons can greatly affect electrical activities $[21,22]$, and it is believed that the probabilistic gating of voltage-dependent ion channels is a source of electrical channel noise in neurons [21]. The collective electrical behavior of neurons is regulated by the size of the membrane patch or the channel noise. A potential mechanism could be coherence resonance-like behavior induced by internal noise. Schmid et al. [22] reported that capacitance fluctuations caused channel noise reduction in stochastic HodgkinHuxley systems. Fox et al. [25] used the autocorrelation functions of the channel noise to measure the effect of channel noise. Ozer et al. [26] suggested that channel blocking was effective in controlling the regularity of spontaneous spiking in Newman-Watts networks of HodgkinHuxley neurons. Sun et al. [27] investigated the effects of channel noise on firing coherence in small-world HodgkinHuxley neuronal networks. Gong et al. [28,29] simulated the effect of channel block on the collective spiking activity of coupled stochastic Hodgkin-Huxley neurons and random networks.

Some previous studies have discussed the effect of ion channel block on the excitability of neurons and collective electrical behavior of neurons in networks. For example, Uchitel [30] investigated the effects of toxins affecting neuronal calcium channels. Johnson et al. [31] studied the roles of drug and channel structure in $\mathrm{Ca}^{2+}$ channel block by phenylalkylamines. Schmid et al. [32] discussed the effect of channel block on the spiking activity of excitable membranes in a stochastic Hodgkin-Huxley model.

In this paper, we focus on ion channel block-induced breakup of spiral waves in a two-dimensional square array, in which Hodgkin-Huxley neurons are connected in a small-world network. The factors $x_{\mathrm{K}}$ and $x_{\mathrm{Na}}$ are defined to describe the fraction of unblocked (active) potassium and sodium channels, respectively. A smaller value of $x_{\mathrm{K}}$ or $x_{\mathrm{Na}}$ is approached when channels are poisoned deeply. Based on mean field theory, a statistical variable is also defined, to allow detection of the critical condition for breakup of spiral waves induced by ion channel block in neurons.

\section{Mathematical model and discussion}

The dynamics of the membrane potential of Hodgkin-
Huxley neurons in a small-world network, and the stochastic properties of this model [25], are described by the following equations:

$$
\begin{aligned}
& C_{m} \frac{\mathrm{d} V_{i j}}{\mathrm{~d} t}= \tilde{g}_{\mathrm{K}} n_{i j}^{4}\left(V_{\mathrm{K}}-V_{i j}\right)+\tilde{g}_{\mathrm{Na}} m_{i j}^{3} h_{i j}\left(V_{\mathrm{Na}}-V_{i j}\right) \\
&+\tilde{g}_{\mathrm{L}}\left(V_{\mathrm{L}}-V_{i j}\right)+I_{i j}+D \sum_{k l} \varepsilon_{i j k l}\left(V_{k l}-V_{i j}\right), \\
& \frac{\mathrm{d} y_{i j}}{\mathrm{~d} t}= \alpha_{y}\left(V_{i j}\right)\left(1-y_{i j}\right)-\beta_{y}\left(V_{i j}\right) y_{i j}(y=m, h, n), \\
& \alpha_{m}=a_{m}\left(V_{i j}\right)=\frac{0.1\left(V_{i j}+40\right)}{1-\exp \left(-\left(V_{i j}+40\right) / 10\right)}, \\
& \beta_{m}=\beta_{m}\left(V_{i j}\right)=4 \exp \left(-\left(V_{i j}+65\right) / 18\right), \\
& \alpha_{h}=\alpha_{h}\left(V_{i j}\right)=0.07 \exp \left(-\left(V_{i j}+65\right) / 20\right), \\
& \beta_{h}=\beta_{h}\left(V_{i j}\right)=\frac{1}{1+\exp \left(-\left(V_{i j}+35\right) / 10\right)}, \\
& \alpha_{n}=\alpha_{n}\left(V_{i j}\right)=\frac{0.01\left(V_{i j}+55\right)}{1-\exp \left(-\left(V_{i j}+55\right) / 10\right)}, \\
& \beta_{n}=\beta_{n}\left(V_{i j}\right)=0.125 \exp \left(-\left(V_{i j}+65\right) / 80\right) .
\end{aligned}
$$

The integer subscripts $i$ and $j$ denote the position of neurons in the two-dimensional small-world network. The variables $V, m, n$, and $h$ describe the membrane potential and gating parameters of neuronal ion channels. The membrane capacitance is $C_{m}=1 \mu \mathrm{F} / \mathrm{cm}^{2}$. The maximal potassium conductance is $\tilde{g}_{\mathrm{K}}=36 \mathrm{mS} / \mathrm{cm}^{2}$, the maximal sodium conductance is $\tilde{g}_{\mathrm{Na}}=120 \mathrm{mS} / \mathrm{cm}^{2}$, the leakage current conductance is $\tilde{g}_{\mathrm{L}}=0.3 \mathrm{mS} / \mathrm{cm}^{2}$, and the external injection current is $I_{i j}=0$. The reversal potentials were $V_{\mathrm{K}}=-77 \mathrm{mV}$, $V_{\mathrm{Na}}=50 \mathrm{mV}$, and $V_{\mathrm{L}}=-54.4 \mathrm{mV} . D$ is the intensity of coupling, and $\varepsilon_{k l i j}$ describes the connection state (on or off) between sites $(k, l)$ and $(i, j) . \varepsilon_{k l i j}=1$ if site $(k, l)$ is connected with site $(i, j)$; otherwise, $\varepsilon_{k l i j}=0 . \xi_{m}(t), \xi_{h}(t)$, and $\xi_{n}(t)$ are independent Gaussian white noise sources and their statistical properties are described by the following equations:

$$
\begin{aligned}
\left\langle\xi_{m}(t)\right\rangle & =0,\left\langle\xi_{m}(t) \xi_{m}\left(t^{\prime}\right)\right\rangle=\frac{2 \alpha_{m} \beta_{m}}{N_{\mathrm{Na}}\left(\alpha_{m}+\beta_{m}\right)} \delta\left(t-t^{\prime}\right) \\
& =D_{m} \delta\left(t-t^{\prime}\right), \\
\left\langle\xi_{n}(t)\right\rangle & =0,\left\langle\xi_{n}(t) \xi_{n}\left(t^{\prime}\right)\right\rangle=\frac{2 \alpha_{n} \beta_{n}}{N_{\mathrm{K}}\left(\alpha_{n}+\beta_{n}\right)} \delta\left(t-t^{\prime}\right) \\
& =D_{n} \delta\left(t-t^{\prime}\right), \\
\left\langle\xi_{h}(t)\right\rangle & =0,\left\langle\xi_{h}(t) \xi_{h}\left(t^{\prime}\right)\right\rangle=\frac{2 \alpha_{h} \beta_{h}}{N_{\mathrm{Na}}\left(\alpha_{h}+\beta_{h}\right)} \delta\left(t-t^{\prime}\right) \\
& =D_{h} \delta\left(t-t^{\prime}\right) .
\end{aligned}
$$

$D_{m}, D_{n}$, and $D_{h}$ describe the noise intensity. The function $\delta\left(t-t^{\prime}\right)=1$ at $t=t^{\prime}$, and $\delta\left(t-t^{\prime}\right)=0$ at $t \neq t^{\prime} . N_{\mathrm{Na}}$ and $N_{\mathrm{K}}$ are the total numbers of sodium and potassium channels, respectively, present in a given membrane patch. In the case of 
homogeneous ion channel density, $\rho_{\mathrm{Na}}=60 \mu \mathrm{m}^{-2}$ and $\rho_{\mathrm{K}}=18$ $\mu \mathrm{m}^{-2}$. The total channel number is decided by $N_{\mathrm{Na}}=\rho_{\mathrm{Na}} s$ and $N_{\mathrm{K}}=\rho_{\mathrm{K}} s$, where $s$ describes the size of the membrane patch. As reported in [32], the influence of channel block on the excitability of a membrane patch of a certain size is twofold: The conductance is changed by the change in channel density, and the channel noise is increased by the downregulation of working ion channels. The change in conductance is measured by

$$
\bar{g}_{\mathrm{K}}=\bar{g}_{\mathrm{K}}^{\max } x_{\mathrm{K}}, \bar{g}_{\mathrm{Na}}=\bar{g}_{\mathrm{Na}}^{\max } x_{\mathrm{Na}},
$$

where $x_{\mathrm{K}}$ and $x_{\mathrm{Na}}$ denote the ratios of active ion channels to the total number of potassium $\left(N_{\mathrm{K}}\right)$ or sodium $\left(N_{\mathrm{Na}}\right)$ channels, respectively. In the numerical studies, the values for $x_{\mathrm{K}}$ and $x_{\mathrm{Na}}$ are set between 0 and 1 , where a higher ratio represents a larger proportion of active channels. The statistical properties [32] of the channel noise [25] are defined by

$$
\begin{aligned}
\left\langle\xi_{m}(t)\right\rangle= & 0,\left\langle\xi_{m}(t) \xi_{m}\left(t^{\prime}\right)\right\rangle=\frac{2 \alpha_{m} \beta_{m}}{N_{\mathrm{Na}} x_{\mathrm{Na}}\left(\alpha_{m}+\beta_{m}\right)} \delta\left(t-t^{\prime}\right) \\
= & D_{m} \delta\left(t-t^{\prime}\right), \\
\left\langle\xi_{n}(t)\right\rangle= & 0,\left\langle\xi_{n}(t) \xi_{n}\left(t^{\prime}\right)\right\rangle=\frac{2 \alpha_{n} \beta_{n}}{N_{\mathrm{K}} x_{\mathrm{K}}\left(\alpha_{n}+\beta_{n}\right)} \delta\left(t-t^{\prime}\right) \\
= & D_{n} \delta\left(t-t^{\prime}\right), \\
\left\langle\xi_{h}(t)\right\rangle= & 0,\left\langle\xi_{h}(t) \xi_{h}\left(t^{\prime}\right)\right\rangle \\
= & \frac{2 \alpha_{h} \beta_{h}}{N_{\mathrm{Na}} x_{\mathrm{Na}}\left(\alpha_{h}+\beta_{h}\right)} \delta\left(t-t^{\prime}\right) \\
= & D_{h} \delta\left(t-t^{\prime}\right) .
\end{aligned}
$$

According to these equations, the intensity of channel noise will change when different values for $x_{\mathrm{K}}$ and $x_{\mathrm{Na}}$ are selected. The statistical properties of the measurable variables (e.g. the neuronal membrane potential) are described by

$$
\begin{gathered}
F=\frac{1}{N^{2}} \sum_{j=1}^{N} \sum_{i=1}^{N} V_{i j}=<V>, \\
R=\frac{<F^{2}>-<F>^{2}}{\frac{1}{N^{2}} \sum_{j=1}^{N} \sum_{i=1}^{N}\left(<V_{i j}^{2}>-<V_{i j}>^{2}\right)},
\end{gathered}
$$

where $R$ is a factor of synchronization. A smaller value of $R$ is often associated with the ordered state. The number of neurons or oscillators is $N^{2}$ and the variable $V_{i j}$ is the membrane potential of the neuron at site $(i, j)$. In the next section, the factors of synchronization are plotted as a function of the parameters $x_{\mathrm{K}}$ and $x_{\mathrm{Na}}$, to detect the critical condition for spiral wave breakup induced by block of ion channels.

\section{Numerical results and discussion}

In this section, a well-developed spiral wave in the network is regarded as the initial state, and then channel block (poisoning) is considered. In the numerical studies, the Eulerian difference method and no-flux boundary condition are used, with a time step $h$ of 0.001 , coupling intensity $D=1.0$, and external forcing current $I_{i j}=0$. It is confirmed that a spiral can develop and occupy the whole network, in which 40000 neurons are arranged on the nodes of the $200 \times 200$ array. As reported previously [33], a minimum size of the domain was necessary to support the occurrence of a spiral wave. A network with $200 \times 200$ nodes could reliably simulate the development of a spiral wave. The small-world network can be described by local regular networks and long-range connections with a certain probability $p$. In the following studies, 90000 neurons were located in a two-dimensional array with $300 \times 300$ sites. Extensive numerical studies have shown that a spiral wave can emerge and cover an entire system with low long-range connection probability, and that spiral wave breakup is induced when the long range connection probability exceeds certain thresholds [3]. Our aim is to focus on the effects of variations of conductance, and thus the channel noise-induced transition of the spiral wave. Therefore, a long range connection probability $p$ of 0.02 (which was able to completely support spiral wave survival) is used throughout the study.

As shown in Figure 1, a spiral wave can develop and occupy most of the area in a network with $300 \times 300$ neurons, within a transient period of about 3000 time units. It takes about 15000 time units for the developing spiral wave to occupy the whole network, and the results are similar when the network is constructed by the two-dimensional array with $200 \times 200$ neurons. As a result, the spiral wave in the center of the network is surrounded by the segments near the boundary of networks.

At first, no channel noise is considered, and then each neuron of the network is described by the deterministic Hodgkin-Huxley model. The factor of synchronization is
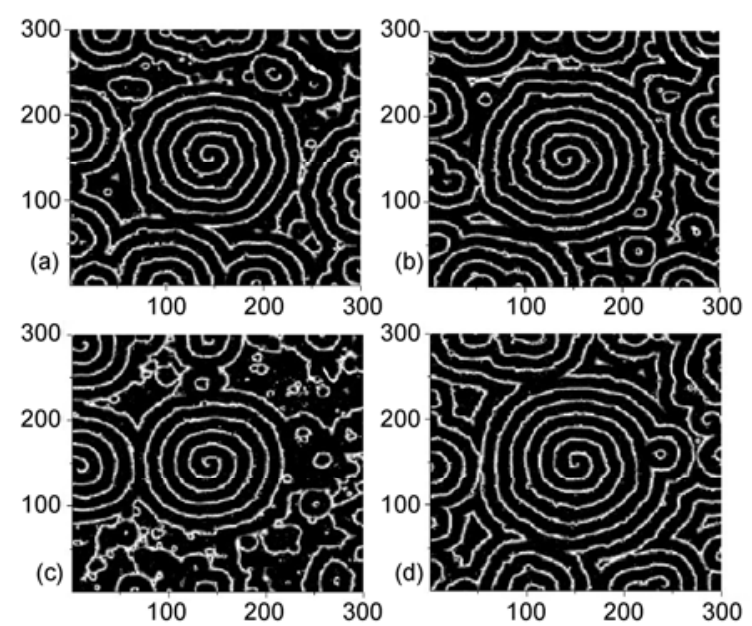

Figure 1 Snapshots of stable rotating spiral waves in the absence and presence of channel noise. (a) Spiral wave without channel noise at 2000 time units; (b) spiral wave without channel noise at 3000 time units; (c) spiral wave in the presence of channel noise $(s=20)$ at 1000 time units; (d) spiral wave in the presence of channel noise at 3000 time units. Long-range probability $p=0.02$; coupling coefficient $D=1$. The snapshots are plotted in grayscale, from black (about $-80 \mathrm{mV}$ ) to white (about $40 \mathrm{mV}$ ). 
plotted as a function of $x_{\mathrm{K}}$ at a fixed $x_{\mathrm{Na}}$ of 1 . The initial state shown in Figure 1(b) is used, and a distinct phase transition is observed. These results can be understood using bifurcation theory [32].

Figure 2 shows that an abrupt decay occurs when the parameter $x_{\mathrm{K}}$ reaches a threshold of about 0.2 . Thus, breakup of the spiral wave occurs when $x_{\mathrm{K}}$ is less than the threshold; otherwise, the spiral wave keeps alive greatly. A potential mechanism could be that channel noise intensity is increased greatly below threshold and the breakup of the spiral wave is induced. The statistical function $R$ measures the collective properties. Spiral wave is an ordered state and a distinct phase transition occurs when spiral wave breakup is induced. Next, snapshots are plotted to show the final state at $t=1000$ time units when the ratio $x_{\mathrm{K}}$ is close to the threshold (Figure 3).

As shown in Figure 3, the spiral wave encounters breakup when the fraction of active potassium channels $x_{\mathrm{K}}$ is close to the threshold of 0.2 , whereas the spiral wave stays robust above the threshold. Next, variation of $x_{\mathrm{Na}}$ is investigated to calculate the distribution of factors of synchronization (Figure 4). The corresponding snapshots are also plotted to illustrate the breakup of the spiral wave (Figure 5).

Figure 4 shows that the spiral wave encounters breakup when the fraction of active sodium channels $x_{\mathrm{Na}}$ is close to a threshold of about 0.4 , whereas the spiral wave is robust above this threshold. When $x_{\mathrm{Na}}$ is set to 0.3 , the spiral wave disappears and the network activity becomes homogeneous (Figure 5).

Figure 5 illustrates that the spiral wave transitions into different states (broken segments or homogeneous states) when $x_{\mathrm{Na}}$ decreases below the threshold. Comparison of the results in Figures 2 and 3 with Figures 4 and 5 raises some questions for discussion. For both potassium and sodium channels, a distinct decline in the factor of synchronization is seen with increasing values of $x_{\mathrm{Na}}\left(x_{\mathrm{K}}\right)$. Schmid et al. [32] reported that channel block can affect the spiking activity of excitable membranes in a stochastic Hodgkin-Huxley model.

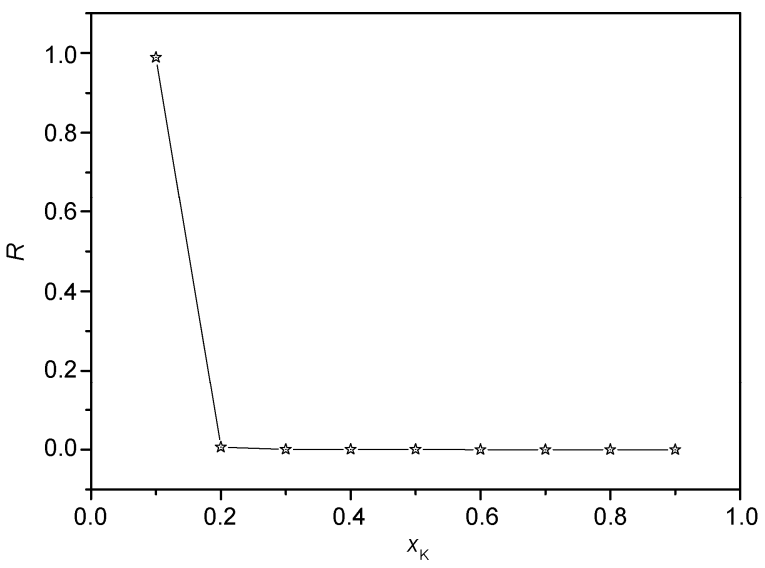

Figure 2 The distribution of factors of synchronization $(R)$ for different values of $x_{\mathrm{K}}$. The transient period is about 1000 time units and $x_{\mathrm{Na}}$ is fixed at 1 .

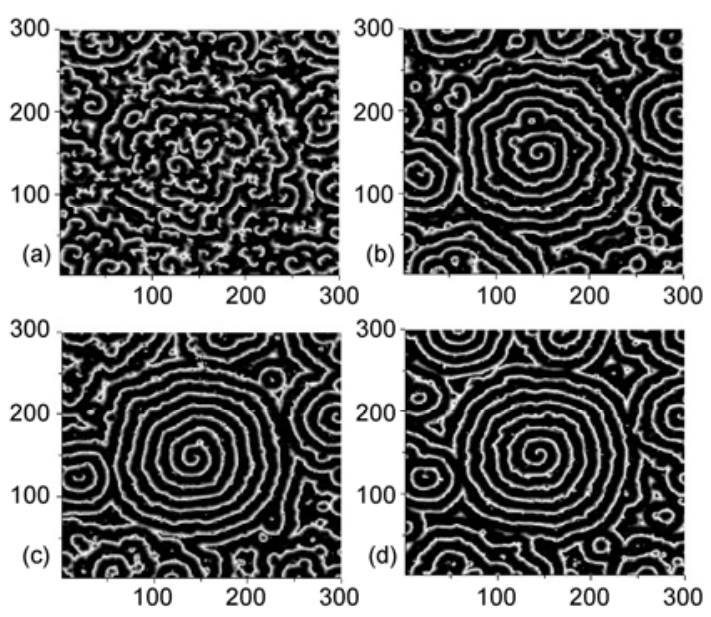

Figure 3 Snapshots of spiral waves under different degrees of potassium channel block at $t=1000$ time units. (a) $x_{\mathrm{K}}=0.2$; (b) $x_{\mathrm{K}}=0.3$; (c) $x_{\mathrm{K}}=0.4$; (d) $x_{\mathrm{K}}=0.5$. The snapshots are plotted in grayscale from black (about $-80 \mathrm{mV}$ ) to white (about $50 \mathrm{mV}$ ). The coupling coefficient $D=1$.

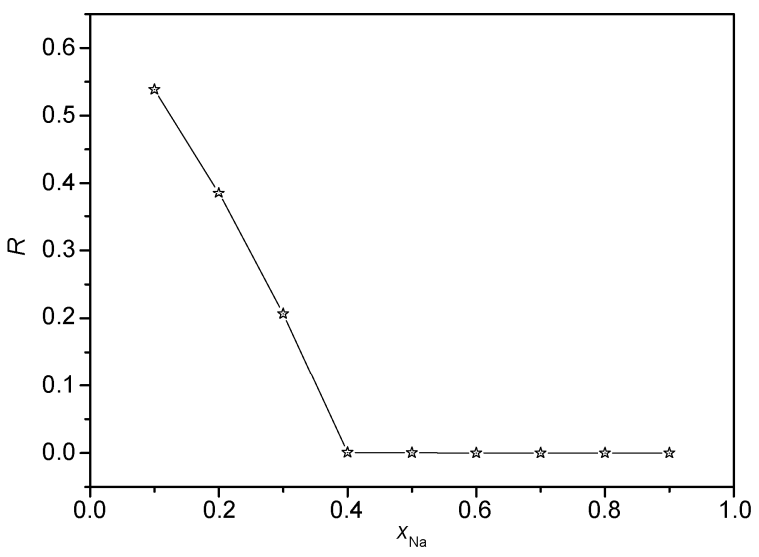

Figure 4 The distribution of factors of synchronization for different values of $x_{\mathrm{Na}}$. The transient period is about 1000 time units and $x_{\mathrm{K}}$ is $\mathrm{f}$ ixed at 1 .
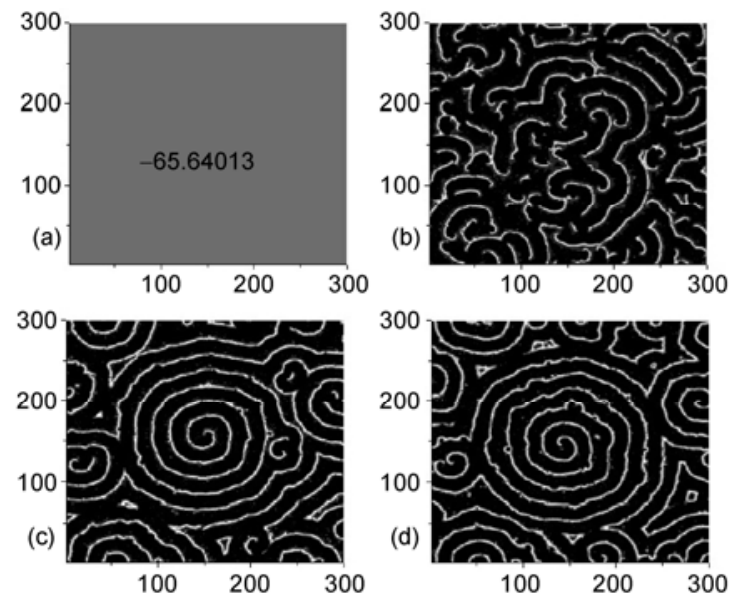

Figure 5 Snapshots showing breakup of spiral waves due to sodium channel block at $t=1000$ time units. (a) $x_{\mathrm{Na}}=0.3$; (b) $x_{\mathrm{Na}}=0.4$; (c) $x_{\mathrm{Na}}=0.5$; (d) $x_{\mathrm{Na}}=0.6$. The snapshots are plotted in grayscale from black (about $-80 \mathrm{mV}$ ) to white (about $50 \mathrm{mV}$ ). The coupling coefficient $D=1$. 
It is helpful to understand the mechanism of the decay in the factor of synchronization. A sub-critical Hopf-bifurcation takes place, a stable spiking solution and oscillatory solution arise, and the stable, non-spiking solution becomes unstable. With further reductions in $x_{\mathrm{K}}$, the oscillatory spiking solution encounters instability. As a result, a breakup of the spiral wave occurs, and a distinct phase transition is observed. The block of sodium channels causes only a small, practically negligible, change in the resting membrane potential. Therefore, the spiral wave keeps alive at most values of $x_{\mathrm{Na}}$. Further decreasing $x_{\mathrm{Na}}$ merely decreases the number of sodium channels, enhancing internal channel noise and inducing a breakup of the spiral wave.

Next, we investigate this effect of weak channel noise. In Figure 6 , the distribution of factors of synchronization is plotted to visualize the effect of potassium channel block.

Again, an abrupt decay occurs with increasing $x_{\mathrm{K}}$, at a threshold of about 0.2 . Figure 7 shows the snapshots corresponding to values of $x_{\mathrm{K}}$ close to the threshold, showing breakup of the spiral wave.

The results in Figure 7 show that breakup of the spiral wave occurs when the factor $x_{\mathrm{K}}$ is less than the threshold in the presence of weak channel noise. The spiral wave stays very robust when $x_{\mathrm{K}}$ exceeds the threshold. Comparison of the results in Figures 2 and 3 with Figures 6 and 7 reveals that the decay and phase transition occurs at similar thresholds $\left(x_{K}=0.2\right)$, whereas the peak values of the factors of synchronization are different. A potential cause may be the action of channel noise.

We next test the effects of sodium channel block on spiral waves in the presence of weak channel noise (Figures 8 and 9).

In the presence of weak channel noise, an abrupt decay is observed when $x_{\mathrm{Na}}$ increases to a threshold of about 0.4 . The corresponding snapshots are plotted in Figure 9.

Figure 9 shows that breakup of the spiral wave is also induced when $x_{\mathrm{Na}}$ is less than the threshold. When channel noise is present, any slight shift in $x_{\mathrm{Na}}$ or $x_{\mathrm{K}}$ changes the

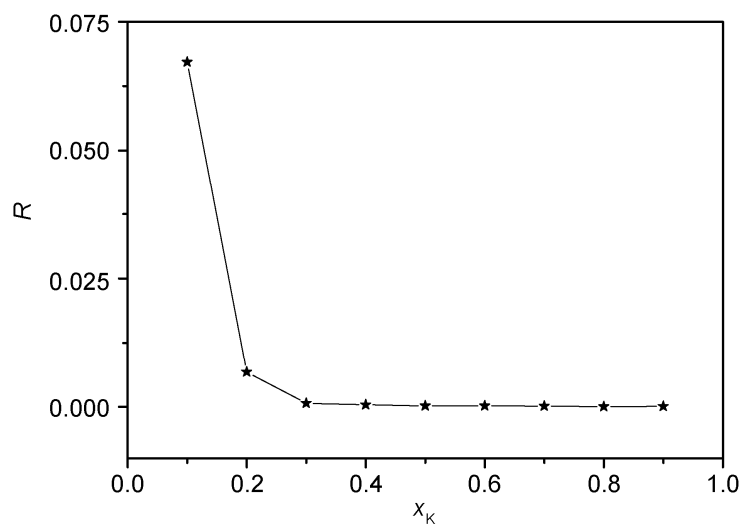

Figure 6 The distribution of factors of synchronization for different values of $x_{\mathrm{K}}$ in the presence of weak channel noise. The transient period is about 1000 time units, the intensity of channel noise is described by membrane patch $s=20$, and $x_{\mathrm{Na}}$ is fixed at 1 in the numerical simulations.
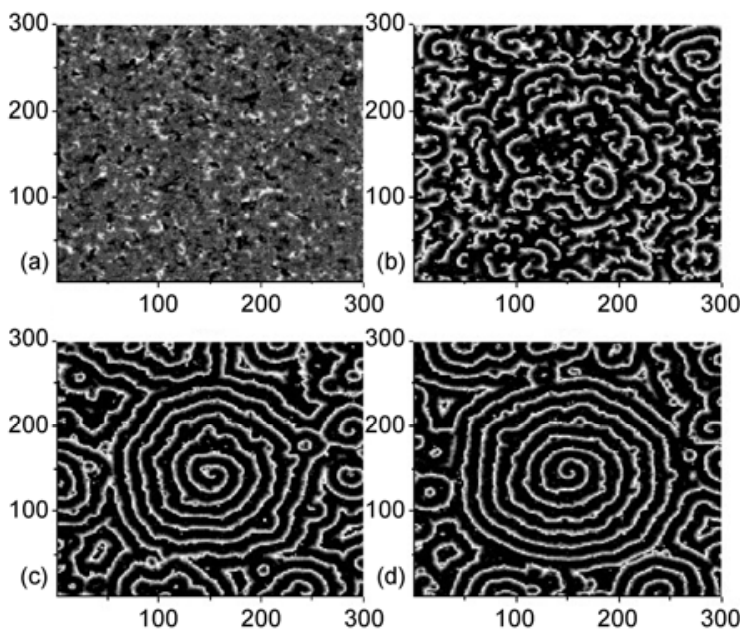

Figure 7 Snapshots showing breakup of spiral wave due to potassium channel block in the presence of weak channel noise, at $t=1000$ time units. (a) $x_{\mathrm{K}}=0.1$; (b) $x_{\mathrm{K}}=0.2$; (c) $x_{\mathrm{K}}=0.3$; (d) $x_{\mathrm{K}}=0.4$. The intensity of channel noise is described by membrane patch $s=20$. The snapshots are plotted in grayscrale from black (about $-80 \mathrm{mV}$ ) to white (about $50 \mathrm{mV}$ ). The coupling coefficient $D=1$.

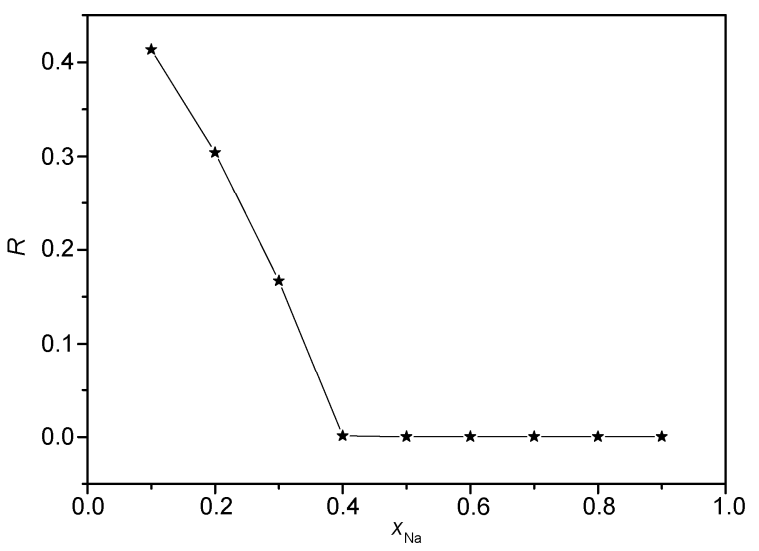

Figure 8 The distribution of factors of synchronization for different values of $x_{\mathrm{Na}}$ in the presence of weak channel noise. The transient period is about 1000 time units and $x_{\mathrm{K}}$ is fixed at 1 in numerical simulation studies. The intensity of channel noise is described by membrane patch $s=20$.

intensity of channel noise greatly, according to eqs. (10)-(12). The distinct decay for factors of synchronization and phase synchronization could result from the change in conductance and decrease in channel noise. The curves in Figures 2, 4, 6, and 8 confirm that a smaller factor of synchronization is often approached, and this is associated with an ordered state in which the spiral wave stays robust despite ion channel block and weak channel noise. Breakup of the spiral wave can be induced by blockade of either sodium or potassium channels. According to the nonlinear terms $\bar{g}_{\mathrm{K}} n_{i j}^{4}\left(V_{\mathrm{K}}-V_{i j}\right), \bar{g}_{\mathrm{Na}} m_{i j}^{3} h_{i j}\left(V_{\mathrm{Na}}-V_{i j}\right)$ in eq. (1), any slight change in sodium or potassium channel conductance can perturb the membrane potential of neurons in different orders of magnitude. Thus, the critical thresholds differ from each other. 

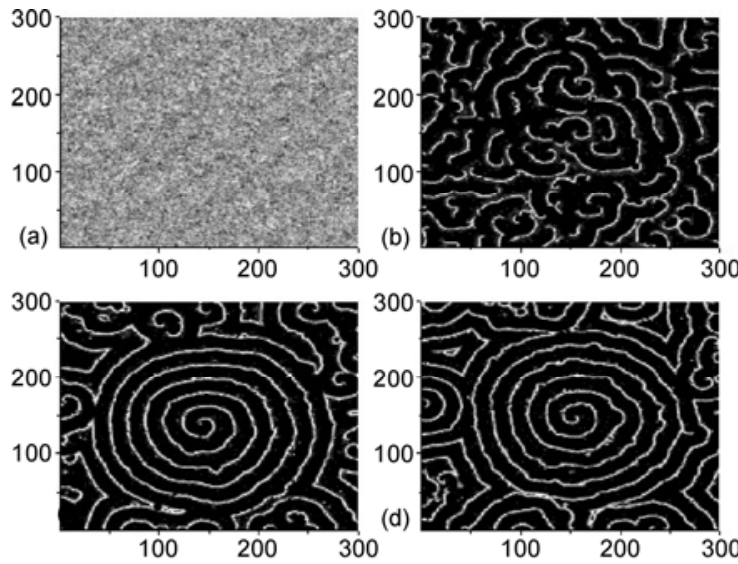

Figure 9 Snapshots showing breakup of spiral waves due to sodium channel block in the presence of weak channel noise at $t=1000$ time units. (a) $x_{\mathrm{Na}}=0.3$; (b) $x_{\mathrm{Na}}=0.4$; (c) $x_{\mathrm{Na}}=0.5$; (d) $x_{\mathrm{Na}}=0.6$. The intensity of channel noise is described by $s=20$. The snapshots are plotted in grayscale from black (about $-80 \mathrm{mV}$ ) to white (about $50 \mathrm{mV}$ ). The coupling coefficient $D=1$.

In addition to the above discussion, some other questions need clarification. The non-flux-boundary condition is used and the robust spiral wave covers most of the area of the network and is surrounded by broken segments. This is not due to the boundary effect, because the initial states (Figure 1(b), (d)) are not perfect spiral waves that cover the whole network of neurons. Spiral waves are a class of ordered waves, and an optimal membrane patch size which internal noise alone induces spontaneous generation of regular action potentials. Channel block can cause breakup of the spiral wave or a disordered state. An interesting question is whether there is a threshold of noise intensity (membrane patch size) that keeps the spiral wave robust under channel blockade. In fact, the results in Figures 6-9 are obtained at fixed channel noise intensity (membrane patch size $s=20$ ), and the critical values of $x_{\mathrm{K}}$ and $x_{\mathrm{Na}}$ can be detected from the curves in Figures 6 and 8. For any fixed values of $x_{\mathrm{K}}$ or $x_{\mathrm{Na}}$, the threshold of noise intensity (membrane patch size) for the robustness of the spiral wave under channel block can be determined by calculating the factors of synchronization $R$ vs. different membrane patch sizes $s$. The distinct changing point in the curve for $R$ vs. $s$ indicates the threshold of the noise intensity that will keep the spiral wave robust under channel blockade.

It is also interesting to investigate these problems using a periodical boundary condition. The two-dimensional square array samples a unit or domain of nervous tissue. It is better to investigate the above problem under periodical boundary conditions, because the neurons close to the boundary of the networks could be affected by the neurons outside the network. In fact, the evolution of spiral waves could be greatly influenced by the degree of channel block and channel noise, while the boundary effect can be neglected because spiral waves in the mammalian cortex are not perfect [19]. Channel noise and/or channel block control the evolution of the spiral wave by adjusting the critical bifurcation parameters, e.g. conductance or density of channels in a membrane patch of a certain size. The dynamics of spiral waves in the network are controlled synchronously and the results could be independent of the boundary condition.

We investigated this problem using periodical boundary conditions (Figure 10).

The results in Figure 10 show that a distinct phase transition of the spiral wave occurs also under periodical boundary conditions. Comparison of the curves in Figure 10(a) with the curve in Figures 6 (and Figure 2, no channel noise), shows that the lines are similar and the critical point is identical. The lines in Figure 10(b) and Figure 8 (and Figure 4, no channel noise) and their critical points are also very similar. This indicates that the statistical factor of synchronization can measure the phase transition of spiral waves well. We also plotted snapshots using near-threshold values of $x_{\mathrm{K}}$ or $x_{\mathrm{Na}}$. This showed that slight differences occur close to the borders of the network, while the center of the media showed similar results to the no-flux boundary condition. To our knowledge, the dynamics of spiral waves mostly depend on the core, or source, although the boundary condition can deform the spiral wave in certain ways. The core of
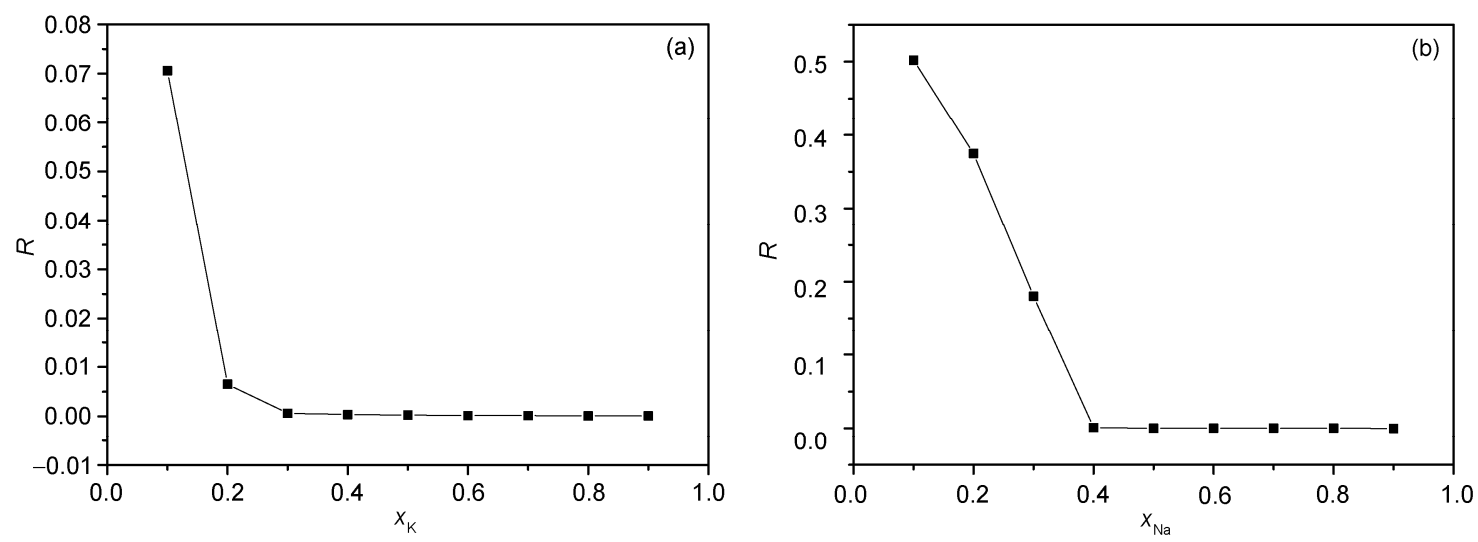

Figure 10 The distribution of factors of synchronization $R$ is plotted for (a) different values of $x_{\mathrm{K}}$ at $x_{\mathrm{Na}}=1$, and (b) different values of $x_{\mathrm{Na}}$ at $x_{\mathrm{K}}=1$. The transient period is about 1000 time units, the intensity of channel noise is described by membrane patch $s=20$, and periodical boundary conditions are used. 
the spiral wave is changed or completely destroyed during ion channel poisoning, and the boundary effect can be ignored under those conditions. A potential mechanism may be that core is invaded because of ion channel poisoning, and breakup of the spiral wave occurs before the boundary effect propagates to the core of the media. In the case of mild intoxication, the effect of boundary conditions is also reduced because of the self-sustaining properties of the spiral wave.

\section{Conclusions}

In this paper, the breakup of a spiral wave by blockade of sodium and potassium channels in a small-world network of Hodgkin-Huxley neurons is investigated in detail. A statistical variable is defined to detect the thresholds of the factors $x_{\mathrm{K}}$ and $x_{\mathrm{Na}}$, which represent the ratios of unblocked channels to the overall number of potassium and sodium channels, respectively. Some interesting results are reported and can be summarized as follows: (1) Breakup of a spiral wave can be induced by block of either sodium or potassium channels, and the critical threshold of $x_{\mathrm{K}}$ and $x_{\mathrm{Na}}$ can be detected from the abrupt decay points in plots of factors of synchronization vs. $x_{\mathrm{K}}$ and $x_{\mathrm{Na}}$. (2) The threshold of factor $x_{\mathrm{Na}}$ is higher the factor $x_{\mathrm{K}}$, which may be related to the fact that sodium and potassium channels affect the membrane potential in different ways, and potassium channels are more important in controlling the neuronal membrane potential. (3) The network activity becomes homogenous and synchronous when sodium channel conductance decreases below the threshold in the absence of channel noise. The reason could be that fewer active ion channels are available because of severe ion channel block, terminating membrane potential fluctuations. (4) The spiral wave stays robust in the presence of weak channel noise, and the spiral wave covers a larger area of the network. It also keeps its robustness against the turbulent state outside, in the case of mild intoxication. (5) The ordered spiral wave provides an effective way to propagate electrical signals between neurons in the quiescent state, and it is resistant against block of certain ion channels. This could explain why animals often maintain normal activities in the case of mild intoxication, whereas severe poisoning can cause a comatose state.

We would like to give great thanks to Dr. Zhang Guoyong and Dr. Jin Wuyin for useful discussions. We also would like to give sincere thanks to the anonymous reviewers for their instructive and straightforward comments, which were very helpful for our forthcoming studies in this field. This work was supported in part by the Educational Tutors Fund Projects of Gansu Province (1010ZTC088), the National Natural Science Foundation of China (11072099) and the Natural Science Foundation of Lanzhou University of Technology (Q200706).

1 He D H, Hu G, Zhan M, et al. Pattern formation of spiral waves in an inhomogeneous medium with small-world connections. Phys Rev E,
2002, 65: 055204

2 Perc M. Effects of small-world connectivity on noise-induced temporal and spatial order in neural media. Chaos Solitons Fractals, 2007, 31: $280-291$

3 Sinha S, Saramäki J, Kaski K. Emergence of self-sustained patterns in small-world excitable media. Phys Rev E, 2007, 76: 015101(R)

4 Wei D Q, Luo X S, Zou Y L. Coupling strength and system size induce firing activity of globally coupled neural network. Commun Theor Phys, 2007, 50: 267-270

5 Jr R E, Brunnet L G. Multistability in networks of Hindmarsh-Rose neurons. Phys Rev E, 2008, 78: 061917

6 Weber S, Hütt M T, Porto M. Pattern formation and efficiency of reaction-diffusion processes on complex networks. Europhys Lett, 2008, 83: 28003

7 Zheng Z G, Feng X Q, Ao B, et al. Synchronization of groups of coupled oscillators with sparse connections. Europhys Lett, 2009, 87: 50006

8 Ma J, Zhang A H, Tang J, et al. Collective behaviors of spiral wave in the networks of Hodgkin-Huxley neurons in presence of channel noise. J Biol Syst, 2010, 18: 243-259

9 Ma J, Tang J, Zhang A H, et al. Robustness and breakup of spiral wave in a two-dimensional lattice networks of neurons. Sci China Phys Mech Astron, 2010, 53: 672-679

10 Ma J, Yang L J, Wu Y, et al. Spiral wave in small-world networks of Hodgkin-Huxley neurons. Commun Theor Phys, 2010, 54: 583-588

11 Gosak M, Marhl M, Perc M. Pacemaker-guided noise-induced spatial periodicity in excitable media. Physica D, 2009, 238: 506-515

12 Chen J X, Hu B B. Spiral breakup and consequent patterns induced by strong polarized advective field. Europhys Lett, 2008, 84: 34002

13 Luo J M, Zhan M. Electric-field-induced wave groupings of spiral waves with oscillatory dispersion relation. Phys Rev E, 2008, 78: 016214

14 Tang G N, Deng M Y, Hu G. Active and passive control of spiral turbulence in excitable media. Phys Rev E, 2008, 77: 046217

15 Yang J Z, Zhang M. Drift of spiral waves in complex GinzburgLandau equation. Commun Theor Phys, 2006, 45: 647-652

16 Ma J, Jin W Y, Yi M, et al. Control of spiral wave and turbulence in the time-varied reaction-diffusion system. Acta Phys Sin, 2008, 57: 2832-2841

17 Samie F H, Josem J. Mechanisms underlying ventricular tachycardia and its transition to ventricular fibrillation in the structurally normal heart. Cardiovasc Res, 2001, 50: 242-250

18 Huang X Y, Troy W C, Yang Q C, et al. Spiral waves in disinhibited mammalian cortex. J Neurosci, 2004, 24: 9897

19 Schiff S J, Huang X Y, Wu J Y. Dynamical evolution of spatiotemporal patterns in mammalian middle cortex. Phys Rev Lett, 2007, 98 : 178102

20 Hodgkin A L, Huxley A F. A quantitative description of membrane current and its application to conduction and excitation in nerve. $\mathrm{J}$ Physiol, 1952, 117: 500-544

21 White J A, Rubinstein J T, Kay A R. Channel noise in neurons. Trends Neurosci, 2000, 23: 131-137

22 Schmid G, Goychuk I, Hänggi P. Capacitance fluctuations causing channel noise reduction in stochastic Hodgkin-Huxley systems. Phys Biol, 2006, 3: 248-254

23 Wang Q Y, Perc M, Chen G R, et al. Delay-enhanced coherence of spiral waves in noisy Hodgkin-Huxley neuronal networks. Phys Lett A, 2008, 372: 5681-5687

24 Perc M. Stochastic resonance on excitable small-world networks via a pacemaker. Phys Rev E, 2007, 76: 066203

25 Fox R F, Lu Y N. Emergent collective behavior in large numbers of globally coupled independently stochastic ion channels. Phys Rev E, 1994, 49: 3421-3431

26 Ozer M, Perc M, Uzuntarla M. Controlling the spontaneous spiking regularity via channel blocking on Newman-Watts networks of HodgkinHuxley neurons. Europhys Lett, 2009, 86: 40008

27 Sun X J, Lei J Z, Perc M, et al. Effects of channel noise on firing coherence of small-world Hodgkin-Huxley neuronal networks. Euro Phys J B, 2011, 79: 61-66 
28 Gong Y B, Xu B, Ma X G, et al. Effect of channel block on the collective spiking activity of coupled stochastic Hodgkin-Huxley neurons. Sci China Ser B-Chem, 2008, 51: 341-346

29 Gong Y B, Hao Y H, Xie Y H, et al. Channel block-optimized spiking activity of Hodgkin-Huxley neurons on random networks. Physica A, 2010, 389: 349-357

30 Uchitel O D. Toxins affecting calcium channels in neurons. Toxicon, 1997, 35: 1161-1191

31 Johnson B D, Hockerman G H, Scheuer T, et al. Distinct effects of mutations in transmembrane segment IVS6 on block of L-type calcium channels by structurally similar phenylalkylamines. Mol Pharmacol, 1996, 50: 1388-1400

32 Schmid G, Goychuk I, Hänggi P. Effect of channel block on the spiking activity of excitable membranes in a stochastic HodgkinHuxley model. Phys Biol, 2004, 1: 61-66

33 Yang $\mathrm{J}$ Z, Zhang $\mathrm{M}$. The investigation of the minimum size of the domain supporting a spiral wave in oscillatory media. Phys Lett A, 2006, 352: 69-72

Open Access This article is distributed under the terms of the Creative Commons Attribution License which permits any use, distribution, and reproduction in any medium, provided the original author(s) and source are credited. 